\title{
Allergic asthma: an overview of metabolomic strategies leading to the identification of biomarkers in the field
}

\author{
A. Villaseñor ${ }^{1}$, D. Rosace ${ }^{1}$, D. Obeso ${ }^{1,2}$, M. Pérez-Gordo ${ }^{2,3}$, T. Chivato $^{3}$, C. Barbas ${ }^{2}$, D. Barber ${ }^{1}$ and M. M. Escribese ${ }^{1,3}$ (iD \\ ${ }^{1}$ Faculty of Medicine, Institute of Applied Molecular Medicine (IMMA), CEU San Pablo University, ${ }^{2}$ Faculty of Pharmacy, Centre for Metabolomics and \\ Bioanalysis (CEMBIO), CEU San Pablo University, and ${ }^{3}$ Basic Medical Sciences Department, Faculty of Medicine, CEU San Pablo University, Boadilla del \\ Monte, Madrid, Spain
}

\section{Clinical \\ \&t Experimental Allergy}

Correspondence:

María M. Escribese, Basic Medical

Sciences Department, Faculty of

Medicine, Institute of Applied

Molecular Medicine (IMMA), CEU San

Pablo University, Campus

Montepríncipe, Crtra. Boadilla del

Monte km 5.3, CP 28668 Boadilla del

Monte, Madrid, Spain.

E-mail:

mariamarta.escribesealonso@ceu.es Cite this as: A. Villaseñor, D. Rosace,

D. Obeso, M. Pérez-Gordo, T. Chivato,

C. Barbas, D. Barber and M. M.

Escribese, Clinical \&t Experimental

Allergy, 2017 (47) 442-456.

\begin{abstract}
Summary
Allergic asthma is a prominent disease especially during childhood. Indoor allergens, in general, and particularly house dust mites (HDM) are the most prevalent sensitizers associated with allergic asthma. Available data show that 65-130 million people are mite-sensitized world-wide and as many as 50\% of these are asthmatic. In fact, sensitization to HDM in the first years of life can produce devastating effects on pulmonary function leading to asthmatic syndromes that can be fatal. To date, there has been considerable research into the pathological pathways and structural changes associated with allergic asthma. However, limitations related to the disease heterogeneity and a lack of knowledge into its pathophysiology have impeded the generation of valuable data needed to appropriately phenotype patients and, subsequently, treat this disease. Here, we report a systematic and integral analysis of the disease, from airway remodelling to the immune response taking place throughout the disease stages. We present an overview of metabolomics, the management of complex multifactorial diseases through the analysis of all possible metabolites in a biological sample, obtaining a global interpretation of biological systems. Special interest is placed on the challenges to obtain biological samples and the methodological aspects to acquire relevant information, focusing on the identification of novel biomarkers associated with specific phenotypes of allergic asthma. We also present an overview of the metabolites cited in the literature, which have been related to inflammation and immune response in asthma and other allergy-related diseases.
\end{abstract}

\section{House dust mite allergic asthma}

Asthma is a multifactorial, chronic syndrome, which varies over time and involves genetic and environmental interactions. It causes reversible airway obstruction through spasm, inflammation and hypersecretion associated with airway hyperresponsiveness (AHR), infiltration of eosinophils and $\mathrm{CD} 4^{+} \mathrm{T}$ helper (Th) type 2 cells into the airway submucosa and airway epithelial remodelling $[1,2]$. The increased prevalence of asthma can be partly accounted for by profound changes in our environment [3], characterized by a rise in urban air pollution and increased indoor allergen exposure, such as animal dander and mites. Indeed, house dust mites (HDM) are the most prevalent allergens associated with asthma and rhinitis around the world [2]. Among HDM, Pyroglyphidae mites are mainly represented by Dermatophagoides pteronyssinus and Dermatophagoides farinae. Several antigens have been identified with mites of the Dermatophagoides species. Recently, the perennial indoor HDM D. pteronyssinus and their 23 associated allergens of Der $\mathrm{p}$ family were summarized using three official allergen-related websites as the source [4]. The major mite allergens Der p 1 and Der p 2 have the ability to induce asthmatic status through different mechanisms. While Der p 1 is a cysteine protease capable of inducing tissue damage and inflammation by cleaving tight-junction (TJ) proteins ZO-1 and occludin, the protease Der $\mathrm{p}$ 2 displays an allergenic role by mimicking the function of MD-2 in the activation of TLR4. Therefore, Der $p 2$ enhances tissue damage, epithelial remodelling and asthma progression [5]. In the case of Derp 1, its proteolytic cleavage increases epithelial permeability leading to a higher allergen presentation by dendritic cells (DCs) [6]. Additionally, Der p 1 can induce innate and adaptive immune response by binding specific receptors such 
as protease-activated receptors (PAR) present on the surface of epithelial cells (ECs) or mast cells, among others. This is shown by the increased amount of PAR on respiratory ECs and mast cells in patients with asthma [7].

\section{Airway remodelling in allergic asthma}

The airway epithelium is the first physical barrier an aeroallergen encounters, and it seems to be key to understanding genetic and environmental interactions in asthma. In fact, asthma is considered an epithelial disease because defects in the epithelial barrier lead to a higher permeability to environmental factors, such as inhaled allergens and pollutant particles and, consequently, to airway wall remodelling $[8,9]$. During airway remodelling structural changes take place from the epithelium to the adventitia due to repeated cycles of airway injury and repair. The main structural changes observed are loss of barrier integrity, goblet cell metaplasia (hypersecretion of mucus), airway smooth muscle hyperplasia and hypertrophy, and subepithelial fibrosis (abnormal deposition of extracellular matrix components, such as collagen) [10-12].

The respiratory epithelium is a pseudostratified structure composed mainly of columnar ciliated ECs, intermixed with mucus-secreting goblet cells, and a pool of basal cells responsible for epithelial regeneration. The functionality of the epithelial barrier is maintained by the formation of TJs and adhering junctions at the apical end of ECs. In the case of HDM sensitization, proteases disrupt epithelial integrity where Der $\mathrm{p} 1$ breaks epithelial TJs, resulting in increased permeability that allows allergens to reach submucosal tissue and activate antigen-presenting cells (APCs) and innate immune cells (Fig. 1a). In this process proteases can also induce bronchial smooth muscle contraction and proliferation [13], and goblet cell metaplasia occurs with the consequent increment in mucus secretion $[8,10,11,14]$. Airway remodelling research is still quite challenging, as there are no reliable biomarkers intimately associated with this process.

\section{Immune response during allergic asthma}

During allergic asthma, injured ECs produce a set of cytokines that lead to Th2 immunity. There are four main pro-Th2 cytokines: thymic stromal lymphopoietin, granulocyte-macrophage colony-stimulating factor (GM-CSF) and interleukins 25 and 33 (IL-25 and IL-33) $[1,15]$. These cytokines induce DC-mediated Th2 signalling and, subsequently, asthma progression. Moreover, during an allergic response to inhaled allergens in the airways, DCs also play an active role by facilitating interaction of the allergen with IgE attached to FceRI, the high-affınity receptor for IgE [16].
Other APCs, such as macrophages, are present in the lung environment under homoeostatic conditions. They act as a sentinel for cellular defence against respiratory pathogens. In fact, in murine models alveolar macrophage (AM) depletion leads to a high degree of inflammation and an increased production of IgE [17], suggesting an association of AMs with progression of the asthmatic status. Additionally, in patients with asthma, AMs can stimulate $\mathrm{T}$ cells to produce more pro-inflammatory cytokines, such as IL-5, amplifying the allergic inflammatory response $[18,19]$. The airway of patients with asthma has been shown to present an increment in the expression of Th2 cells as well as overexpression of IL-4, IL-13, IL-6 and IL-9 pro-inflammatory cytokines [14] (Fig. 1b). The overexpression of IL-4 and IL-13 induces B cells to produce IgE, activates DCs and AMs, and makes goblet cells increase mucal secretion leading to hyperplasia [20]. IL-13 cytokines can also down-regulate Th17 cells involved in maintenance of the mucosal barrier and pathogen clearance, while IL- 6 secreted by Th2 cells, can selectively block Treg-induced immunosuppression by secreting immune-modulator cytokines such as TGF- $\beta$ and IL-10 [21].

Another relevant population of Th cells in asthma are Th9 cells, which are strongly related to asthma initiation and progression. These cells can produce a high titre of IL-9 cytokine which is involved in different processes such as the production of IgE antibodies, in the increment and stimulation of cell infiltration into the respiratory tract, and also plays an important role in collagen deposition and in the survival of innate lymphocyte cells 2 (ILC2) [22]. Unlike B and T cells, ILC2 are a group of innate immune cells that belong to the lymphoid lineage but are devoid of a specific receptor. Their dysregulation as well as an increase in their number have been reported to be associated with allergy or asthma, as activation of these cells contributes to, and supports, type 2 inflammation, acting synergistically with Th2 cells [23].

Research in this field unequivocally demonstrates that allergic asthma is considered a Th2 disease. However, knowledge into the molecular mechanism of this multifactorial disease is not as complete as for other immune diseases with a significant Th2 component, like diabetes or cancer $[24,25]$.

Other essential immune cells in the development of an allergic response and asthma correspond to the eosinophils and mast cells. In fact, eosinophilia is considered as a histologic feature of asthma and is used as a marker of severity and progression. Another powerful marker for asthma detection and classification is eosinophil cationic protein (ECP). This protein is released following eosinophil degranulation and elevated levels in patients with asthma have been correlated with 


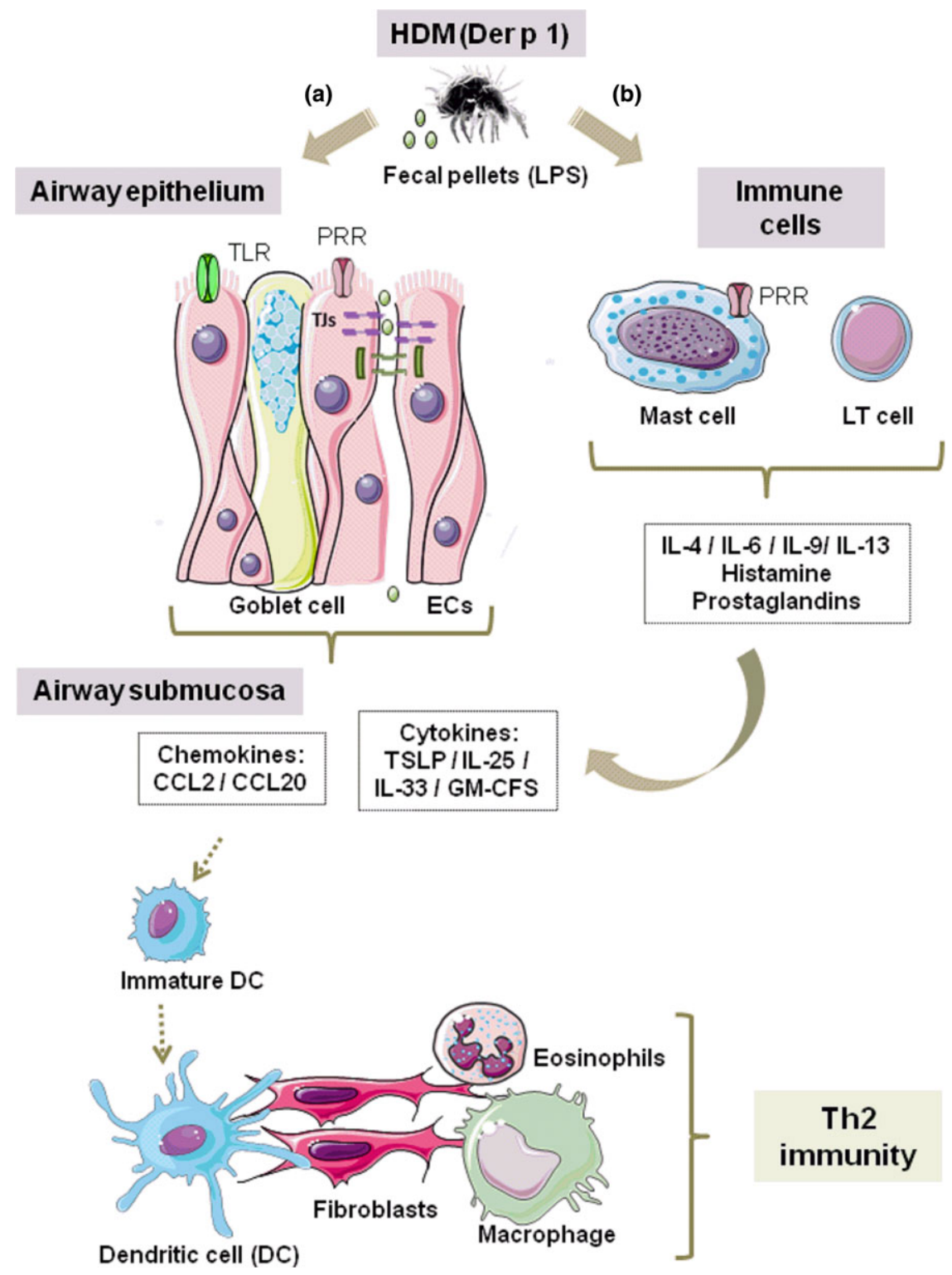

Fig. 1. Effect of HDM allergen Der p 1 and fecal pellet on airway epithelium. (a) Der p 1 protease activity disrupts epithelium integrity, increases permeability and activates pro Th2 cytokines released. Lipopolysaccharide recognition from fecal pellet contributes to EC activation and Th2 immunity. (b) Stimulation of ECs by activation of immune cells also leads to Th2 immunity. LPS, lipopolysaccharide; ECs, epithelial cells; TSLP, thymic stromal lymphopoietin; GM-CSF, granulocyte-macrophage colony-stimulating factor; DC, dendritic cell.

inflammation [26]. Although eosinophilia and ECP levels have been used until now as markers of severity progression in asthma, they are not specific biomarkers for other phenotypes, such as HDM allergic asthma. This is because they are not considered to be reliable, as they do not always classify patients according to phenotype and do not provide a predictive value for treatment efficacy [27].

Mast cells are considered as key cells to instate an allergic response. The number of mast cells and their degranulation are considerably higher in patients with asthma compared to healthy patients. Their main functions in asthma are both secretion of proliferative mediators contained in the granules such as histamine, tryptase and PGD2, and to increase the hyperresponsiveness of airway smooth muscle layer [28]. Moreover, they also participate in airway remodelling through the secretion of mast cell tryptase involved in the growth of ECs and fibroblasts [29, 30].

\section{Clinical biomarkers associated with allergic asthma}

As discussed previously, asthma is influenced by a multitude of factors, although the increasing prevalence of 
asthma and atopic diseases over the last few decades is difficult to explain. Lifestyle factors, environmental exposures and/or interactions between genes and the environment probably play a causal role. Several candidate genes have been associated with asthma outcome and development. This is the case of glutathione $S$-transferases (GSTs), a supergene family implicated in detoxification of reactive oxygen species that appear to be related to asthma susceptibility [31]. Apparently, some mutations of GSTs may be related to asthma onset and AHR, however, contradictory data was found depending on the study group [32, 33]. In fact, Piacentini et al. [32] did not find an association between GSTT, GSTM and GSTP (glutathion S-transferases) gene polymorphisms and the development of asthma in an adult Italian population. While Wang et al. [34] found a correlation between indoor incense burning and GSTs polymorphism in children with asthma, Sohn et al. [34] described a different behaviour of GSTs gene polymorphisms using murine models. Another candidate is $A D A M$ 33, a gene encoding for a disintegrin and metalloprotease glycoprotein in charge of cell-to-cell and cell-to-matrix interaction, which had a higher expression in ECs from patients with asthma than in controls [35].

Furthermore, epigenetic modifications have recently been considered to be important factors in the development of asthma [36, 37]. During the prenatal period, while the development of the airway and immune system, maternal exposure to tobacco smoke, trafficrelated pollutants, viral infection or dust mites have been shown to increase the risk of asthma in offspring [38-41]. The second critical period for asthma onset is throughout early childhood, especially in the first year of life (during alveolar expansion and rebalancing of the immune responses), where severe viral infections in the inferior respiratory tract or the exposure to airborne environmental irritants, HDM allergens and therapeutics (e.g. acetaminophen) have been shown to elevate childhood asthma risk [42-44]. Another marker currently used in medical daily practice to identify severe phenotypes is the fraction of exhaled nitric oxide (NO). However, this does not enable the generation of reliable phenotypes either as it produces contradictory results in predicting eosinophilic airway inflammation [28, 45, 46]. More recently, periostin, a protein observed in adults and children with asthma [47, 48], has been considered a novel biomarker for allergic inflammation progression.

Altogether, the research work performed in the field of allergic inflammation reveals an urgent need to continue looking for early and reliable biomarkers to classify patients and predict therapeutic responses or, in other words, that will define specific phenotypes for allergic asthma. Furthermore, these clinically relevant biomarkers open up new possibilities for novel interventions and the development of preventative therapeutic tools.

\section{Metabolomic approaches to defining allergic phenotypes}

In recent decades, metabolomics has emerged as a new tool to manage complex diseases such as allergy-related conditions, where up to now these specific phenotypes have not been fully characterized and the metabolic changes involved are still largely unknown. Metabolomics works by measuring all possible metabolites in a biological sample, and uses different strategies to pursue this objective. In this sense, working approaches are mainly divided mainly into two strategies: targeted and untargeted metabolomics, each with their own inherent advantages and disadvantages. Independently of the metabolomic approach followed, a reliable outcome will depend on the experimental design and its characteristics. In this context, the type of approach, sample size and type, and analytical platform are described and discussed below.

\section{Metabolomic approach}

Targeted metabolomics is the measurement of defined groups of chemically characterized and biochemically annotated metabolites and is closer to classical hypothesis-based analysis. This approach is essential when a previous knowledge of outstanding metabolites for the disease exists. Meanwhile, untargeted metabolomics is the comprehensive analysis of all the measurable molecules in a sample including chemical unknowns, which may reflect the physiological state of each individual in the study. Due to its comprehensive nature, untargeted metabolomics must be coupled with advanced chemometric techniques, such as multivariate analysis, to reduce the extensive data sets generated into a smaller set of manageable signals. This methodology is highly informative in a discovery phase, working without a prior hypothesis. Untargeted analysis will lead to the identification of novel biomarkers. These can be useful to define patient phenotype, to obtain an accurate diagnosis, to evaluate disease progression or as targets for new pharmacological treatments.

\section{Sample size and type}

Selection of the patients is of paramount importance in multifactorial diseases; in this sense, for example, Chang et al. [49] employed patients with very homogeneous respiratory characteristics compared to controls chosen over respiratory evaluations only. These inclusion criteria enabled the authors to obtain a good modelling of the groups and feasible results after applying non-target analysis. However, for population 
studies the number of patients per group is critical, recommending over 100 samples per group. Herein, Ried et al. [50] targeted more than 100 metabolites in 2925 patients consisting of 147 patients with asthma and 2778 controls. Although the groups were unbalanced, decreasing the value of the statistics, they were able to find some significant differences in asthma in relation with phospholipids. In this quest, for a reliable study, the selection of patients inside the groups must be as homogeneous as possible: same number per group, sex- and age-matched, same body mass index, closest medical illness chart and, if possible, avoid confounder factors such as medications or influential habits, for example smoking or alcoholism.

Sample selection and its manipulation before analysis will define the type of metabolites observed in the study. In the case of respiratory conditions, the difficulty to obtain lung tissue unless working with animal models seems to be a limiting factor. As lung biopsies and bronchoalveolar lavage fluid (BALF) are considered as invasive procedures in humans, a close approximation is the analysis of sputum samples, which is still not recommended by some authors [51, 52]. An alternative option is exhaled breath (EB) condensate, which is a non-invasive sample that has been used in the study of asthma. However, while EB is a relevant sample in respiratory affections because it shows the characteristic compounds from lung metabolism, its greatest handicap is the difficulty to obtain a reliable interpretation and correlation of these metabolites with the disease. In resume, easily extracted samples comprise plasma and urine, which are the two most common biofluids employed in metabolomics, as they are considered to be obtained non-invasively in humans. While plasma represents a global view of the physiological status at the time that the sample is collected, urine represents the end products of metabolism. Therefore, for exploratory studies that are aimed at obtaining a close molecular mechanism of a respiratory disease the use of BALF, lung biopsy, or sputum can be justified. However, when looking for biomarkers with diagnostic or prognostic potential, plasma and urine are the most convenient biofluids.

\section{Analytical platform}

Sample treatment will depend on the kind of compounds of interest and the analytical technique to be employed. Analytical techniques are preferably based on mass spectrometry (MS), usually combined with a separation technique [liquid and gas chromatography (LC, GC) or capillary electrophoresis (CE)] and nuclear magnetic resonance spectroscopy (NMR). Of all the coupled MS techniques, LC-MS has been widely applied and different kinds of samples have been analysed in independent studies such as serum, BALF and lung tissue [53] or EB condensate [54]. From both studies, different classes of compounds have been observed, such as lipids, fatty acids, prostaglandins and purine cycle intermediates. In the case of GC-MS, this technique is suitable for analysing volatile compounds or those chemically volatile after derivatization (e.g. by trimethylsilylation). As expected, GC-MS has been applied to volatile samples such as EB where sample treatment is almost none, although it is also used for urine, BALF and blood samples [55, 56]. The most common types of compounds detected by GC-MS are amino acids, sugars, organic acids and TCA intermediates. Finally, NMR spectroscopy is a non-selective technique considered to be practically universal which requires little or no sample preparation, is unbiased, rapid, robust and quantitative, making it highly suitable for non-target analysis. The principal disadvantage of NMR is its lower sensitivity compared to MS techniques. Regarding NMR-based metabolomics, this has been used to analyse serum, urine and EB condensate from patients with asthma in different projects [57-59].

For respiratory diseases, there has also been an increase in studies and applications that use the electronic nose. This is an instrument developed to recognize all possible volatile components from the breath. This apparatus has been successfully applied in the study of asthma and chronic obstructive pulmonary disease (COPD), showing promising results in the discrimination of groups [56, 60-62]. However, challenges still encountered with this technique including standardization of the breath sample collection, validation of metabolites and, probably, its main bottleneck, the lack of identification of most significant metabolites [63].

The application of each analytical platform characterized by the analytical conditions partially captures section the metabolome and, therefore, does not give a complete picture of the disease. On other hand, despite the significant advances in analytical technologies, biomarker discovery remains a challenge partly because of the overwhelming task of data treatment. The key lies in the ability to distinguish genuine biological variation from analytical and random interferences, although there are now advanced computer tools to tackle this problem. Once the potential biomarkers have been selected there is the arduous task of making the best interpretation of the new findings. This is either based on the bibliographic background or, whenever possible, by projecting the metabolites into known biochemical pathways to create a new biological hypothesis pending verification. This is even harder in multifactorial diseases due to different confounder factors such as medications, age, gender, patients' habits (e.g. smoking), sample size and secondary diseases. 


\section{Metabolomic applications in asthma and allergy-related conditions}

An extensive literature search was performed to look for metabolomic applications related to asthma and allergy-related conditions. The outcome was divided into reviews and research articles, the latter grouped as (i) allergic and (ii) non-allergic/mixed asthma, (iii) food allergy and (iv) anaphylaxis caused in the oral tract (Fig. 2a). In general, the outcome of metabolomics in this field is immature due to the heterogeneity of clinical phenotypes and the complexity of each condition [64-67]. Tentative biomarkers are insufficient to describe the complete underlying processes and the number of published research works is still low $[28,51$, $52,68]$. Hence, a proper validation in a large population and in longitudinal studies is still required. However, reviews of airway diseases remark that although current diagnosis of respiratory diseases performed by clinicians is based on low specific parameters such as medical history, symptoms and clinical blood test, metabolomics is starting to obtain promising findings in the earlier diagnosis, management and understanding of these types of diseases [69-71]. This is even more important in paediatrics where respiratory conditions are the first cause of morbidity and mortality [72]. Regarding to the methodological conditions, pie charts showing the types of biofluids and analytical techniques used in the research articles have been included (Fig. 2b,c). Results showed that blood is the most used biofluid even for respiratory conditions, compared to urine, whereas all high throughput analytical techniques were used throughout equally.

\section{Non-allergic/mixed asthma}

A resume of all the findings published to date on this topic is presented in Table 1. Basically, most studies were carried out in adults (Table 1a), there are two insightful publications into asthma differentiating from COPD (Table 1b) and two studies in children (Table 1c). Concerning adults with asthma, significant advances in metabolic changes linked to the immune response have been reported. In patients with asthma, Jung et al. [73] found decreased levels of arginine in serum, authors correlated this change with the arginine methylation pathway, which is a key process in asthma involved in the regulation of cytokine overexpression. In another study, increased concentrations of some polyunsaturated phospholipids were found to be strongly correlated with asthma risk alleles in a population study which included 147 patients with asthma [50]. Interestingly, in a study with Chinese patients presenting mild persistent asthma, a decrement in inosine level was associated with inflammation and hypoxia [49]. Another study reported increased levels of nicotinamide, adenosine monophosphate and arachidonic acid in plasma as biomarkers of asthmatic inflammation [74]. Besides, authors specifically suggested taurine as a marker of swelling induced by the disease. In another study, Sinha et al. [58] found decreased concentrations of ammonium ion in EB samples, the authors explained this decrement as a reduction in glutaminase activity, an enzyme suppressed by inflammatory cytokines. In two other studies by Loureiro et al. [75, 76] on asthma exacerbation and chronic asthma, authors

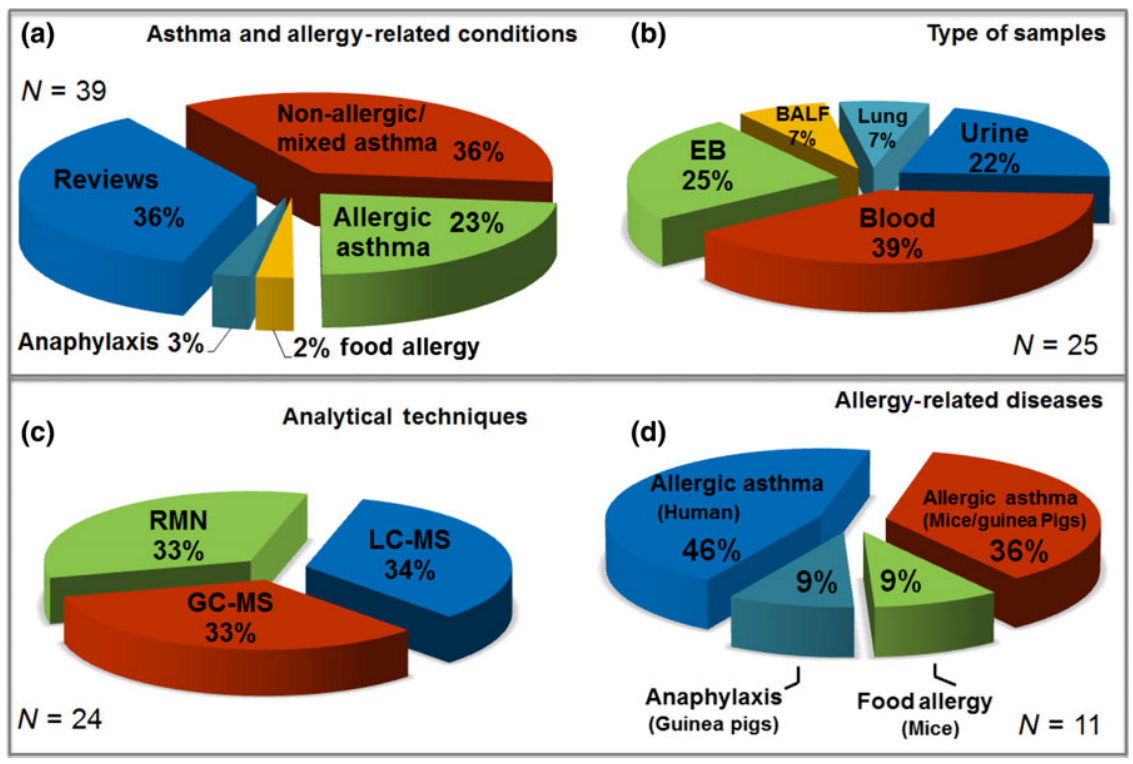

Fig. 2. Percentage pie charts based on publications of (a) asthma and allergy-related conditions, (b) type of samples, (c) analytical techniques and (d) allergy-related diseases. Key: the searching keywords were as follows: 'metabolomics and, asthma, allergy or inflammation' in NCBI PubMed. 


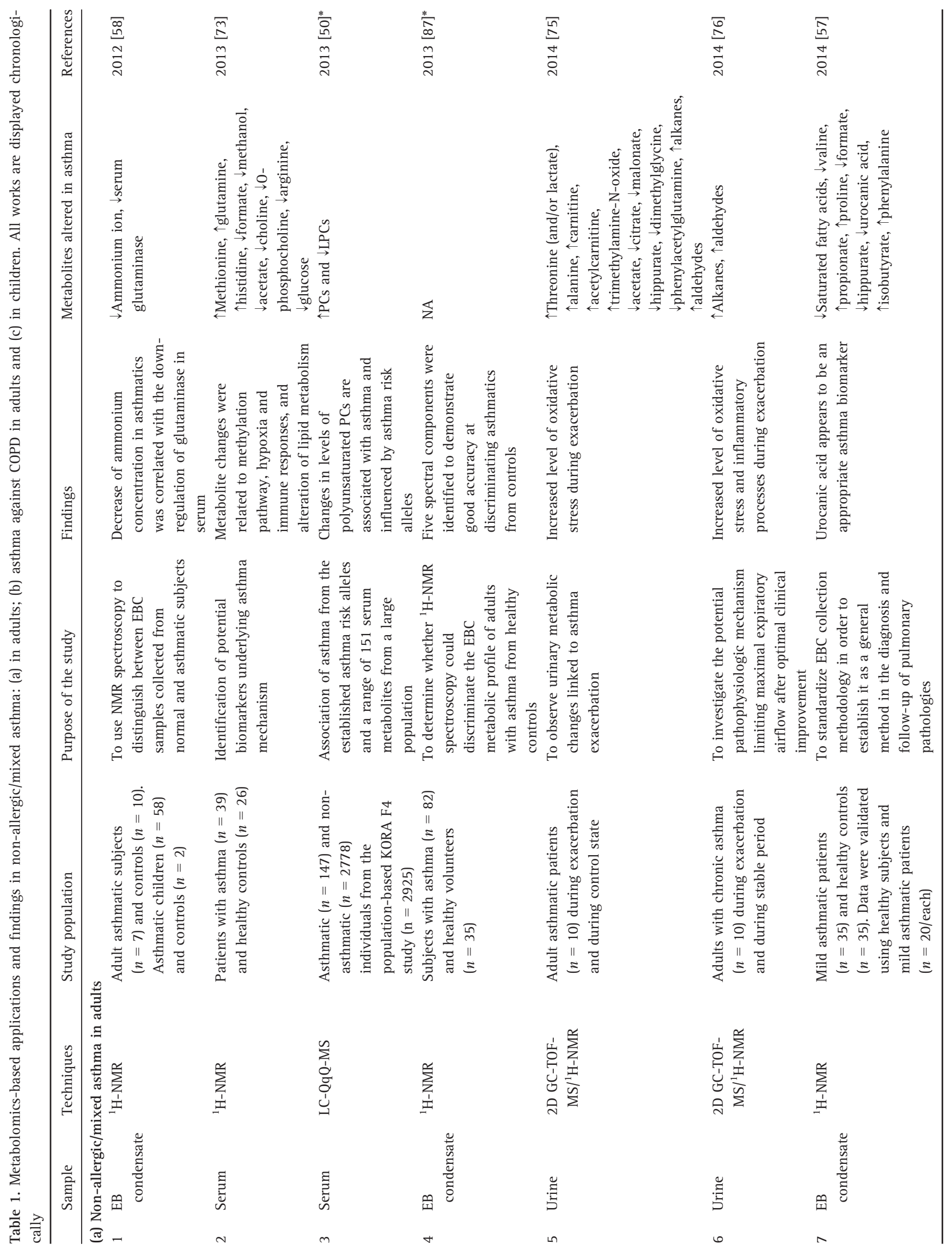




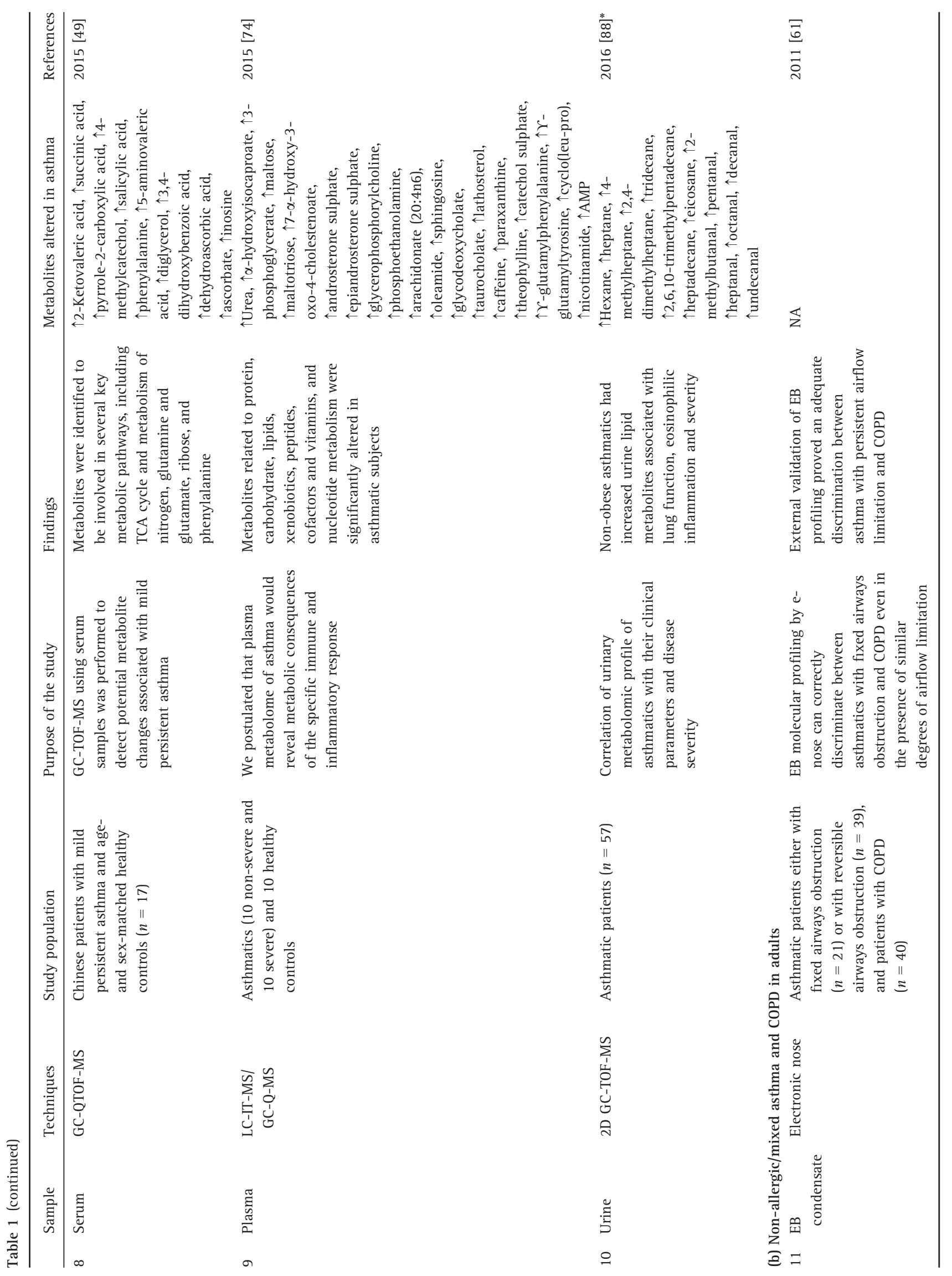




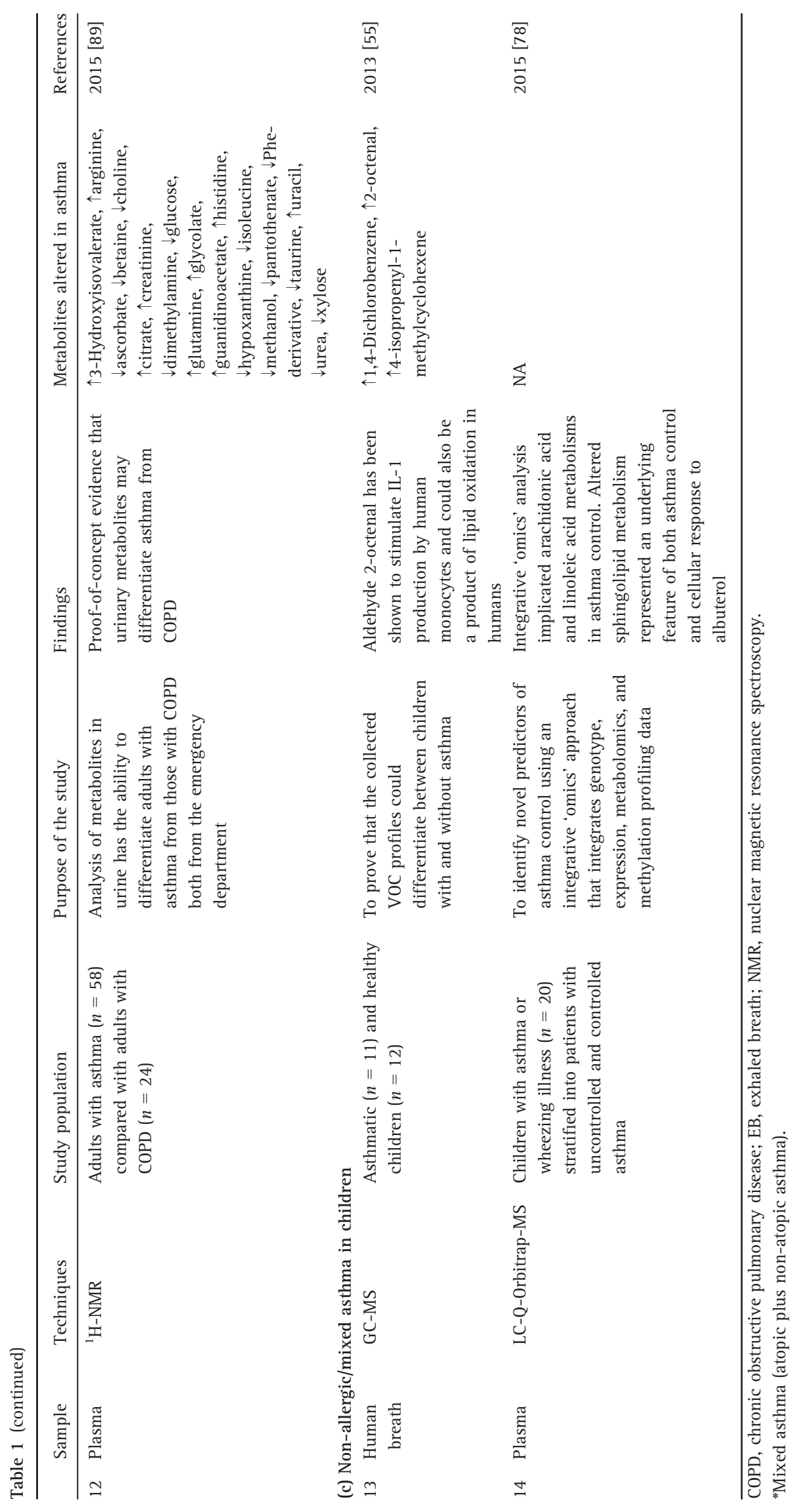




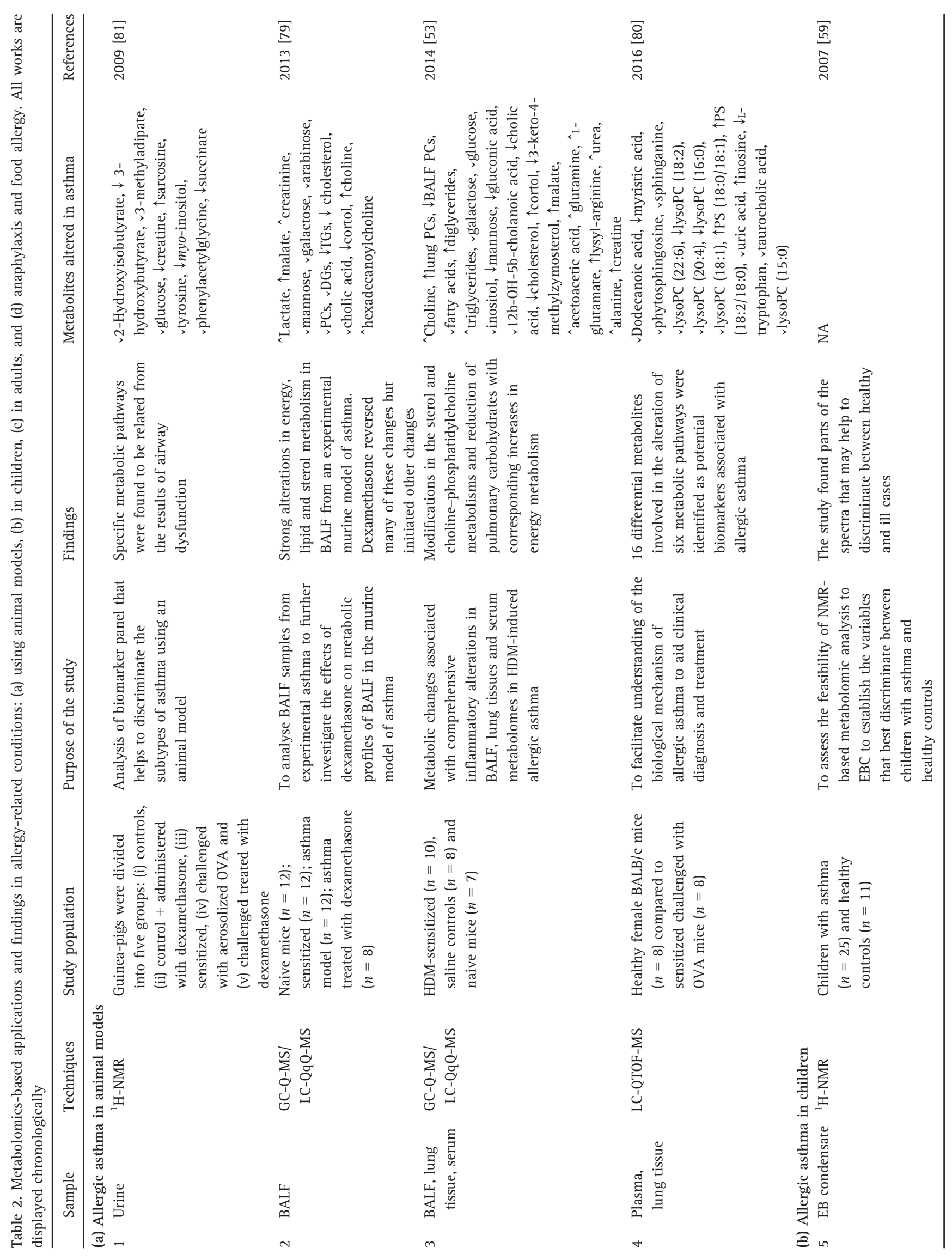




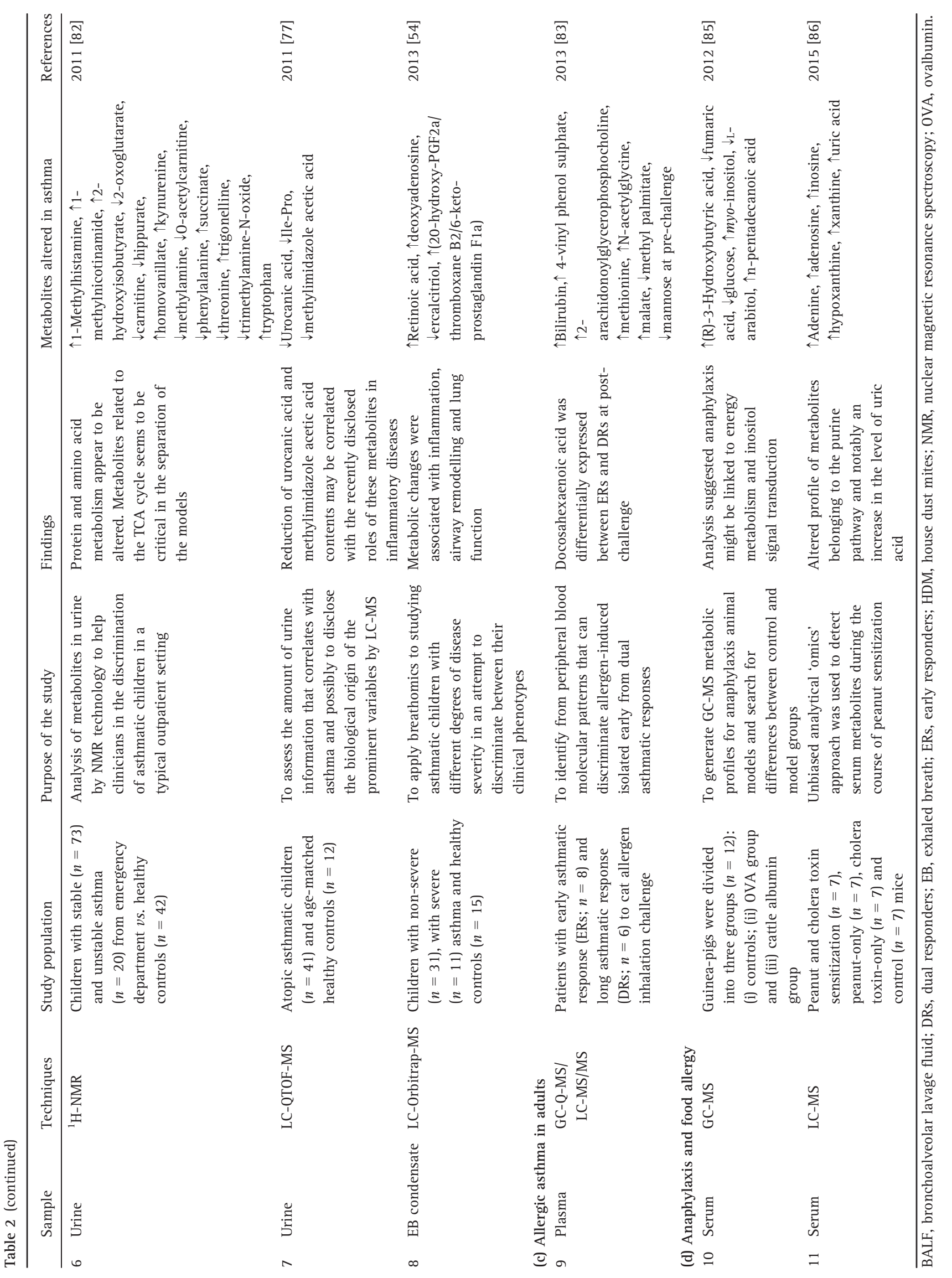


found increased levels of alkanes and aldehydes in both studies, and associated these changes with a higher level of oxidative stress in the worse states of asthma. Furthermore, urocanic acid, a compound from histidine catabolism, was proposed as a potential biomarker of the disease as authors found lower levels in adults with asthma [57]. Interestingly, urocanic acid was also found to be significantly reduced in urine of children with allergic asthma [77]. In paediatric studies with non-allergic asthmatic children an increased level of 2-octenal, a volatile compound, was described to stimulate IL-1 expression [55], whereas in a recent study, sphingolipid metabolism was reported to be altered as an outcome of airway inflammation [78].

\section{Allergy-related diseases}

Regarding research into allergy using metabolomics, the outcome of 11 studies revealed the huge amount of work that remains to be done in this vast field (Fig. $2 \mathrm{~d}$ and Table 2). In allergic asthma, this disease has been studied in animal and human models. In animals, an ovalbumin (OVA)-sensitized mice model showed alterations in the amino acid, energy and lipid metabolic pathways using BALF samples [79]. Whereas when $\mathrm{Yu}$ et al. [80] analysed plasma of the same mice model, authors found significant changes in dodecanoic and myristic acids, phytosphingosine, sphinganine, inosine and taurocholic acid, suggesting these molecules could be involved in the inflammatory response. Another study proposed that a lower level of tyrosine in the urine of guinea-pigs was the result of increased eosinophil and/or neutrophil activity [81]. For HDM allergic asthma, new insights are appearing, herein Ho et al. [53] used a commercial HDM extract to provoke sensitization in mice. Through this approach, pulmonary alterations were observed over the metabolism of lipids and sterols. Along with these changes a significant loss of carbohydrates and the increment in choline in the lung were linked with airway inflammation [51].

In the case of allergic asthma in children, one study suggested retinoic acid as a metabolite related to airway remodelling and inflammation, as it appeared to be elevated in the more severe patients. Likewise, deoxyadenosine, a metabolite of adenosine, was correlated with several pro-inflammatory effects [52]. Additionally, Mattarucchi et al. [77] proposed reduced amounts of urocanic acid, methylimidazole acetic acid and Ile-Pro fragment resembling metabolite as modulating molecules of the immune system in asthma with a role in inflammation. Also, an increased level of 1-methylhistamine in the urine of children with asthma was associated with inflammation [82].
In adults with allergic asthma, a study comparing patients with either a short allergic response (early responders; ERs) or those with a prolonged allergic response (dual responders; DRs), showed a lower level of cortisol in ER patients, assigning it an immunosuppressive and anti-inflammatory role in allergy length response [83, 84]. Interestingly, after an allergic challenge a decrease in docosahexaenoic acid (DHA) was observed in the ER group suggesting the potential of DHA to make the allergic inflammation disappear.

In other types of allergies, a study of anaphylaxis produced by OVA-sensitized guinea-pigs was reported [85]. As expected, high levels of IgE in serum were found in the sensitized model compared to controls, and altered levels of glucose, lipid and inositol phosphate, which were related to the energy pathway and signal transduction. Finally, in food allergy, Kong et al. [86] showed the promising capacities of metabolomics, finding uric acid to be a strong potential biomarker in the accurate prediction of peanut allergy in children. The authors started from a mice model where different metabolites related to purine metabolism were observed and, later, authors validated their measured uric acid in children's urine.

\section{Future directions}

To date, there are no available biomarkers to make a full characterization of allergic asthma phenotypes, which at this moment are poorly characterized within diagnostic criteria in the clinical setting. We are convinced that the search for these biomarkers will improve current treatments of patients by allowing a more precise and personalized medicine. Initial findings have produced promising results in different subtypes of allergy, based mainly on good stratification criteria of the individuals in the studies. However, there is still more work pending in this field. Results found in animals should be correlated in humans and more research must be carried out in human models. Furthermore, there is a need to cover other subtypes of allergy and characterize their phenotypes and specific altered biochemistry using metabolic fingerprinting approach through multiplatform analysis (NMR, LCMS, GC-MS and CE-MS). We expect that in the near future a combination of different markers, including those obtained through metabolomics studies, will give clinicians the opportunity to monitor allergic conditions and predict whether a patient will respond to a specific treatment.

\section{Acknowledgements}

This work was supported by ISCIII (Project number PI15/02256 and PI13/00477), by the Ministry of Economy and Competitiveness (project number 
CTQ2014-55279-R) and by Fundación Mutua Madrileña (AP158912015). Domenico Rosace and David Obeso were supported by FPI-CEU Pre-doctoral Fellowships.

\section{Conflict of interest}

The authors declare no conflict of interest.

\section{References}

1 Lambrecht BN, Leung DY. Initiation and maintenance of allergic inflammation: team work at the interface of innate and adaptive immunity. Curr Opin Immunol 2011; 23:769-71.

2 Gandhi VD, Davidson C, Asaduzzaman M, Nahirney D, Vliagoftis H. House dust mite interactions with airway epithelium: role in allergic airway inflammation. Curr Allergy Asthma Rep 2013; 13:262-70.

3 Thomsen SF. The contribution of twin studies to the understanding of the aetiology of asthma and atopic diseases. Eur Clin Respir J 2015; 2:27803.

4 Gregory LG, Lloyd CM. Orchestrating house dust mite-associated allergy in the lung. Trends Immunol 2011; 32:402-11.

5 Trompette A, Divanovic S, Visintin A et al. Allergenicity resulting from functional mimicry of a Toll-like receptor complex protein. Nature 2009; 457:585-8.

6 Cunningham PT, Elliot CE, Lenzo JC et al. Sensitizing and Th2 adjuvant activity of cysteine protease allergens. Int Arch Allergy Immunol 2012; 158:347-58.

7 Wang JY. The innate immune response in house dust mite-induced allergic inflammation. Allergy Asthma Immunol Res 2013; 5:68-74.

8 Holgate ST. The sentinel role of the airway epithelium in asthma pathogenesis. Immunol Rev 2011; 242:205-19.

9 Gohy ST, Hupin C, Pilette C, Ladjemi MZ. Chronic inflammatory airway diseases: the central role of the epithelium revisited. Clin Exp Allergy 2016; 46:529-42.

10 Chen JC, Chuang JG, Su YY, Chiang BL, Lin YS, Chow LP. The protease allergen Pen c 13 induces allergic airway inflammation and changes in epithelial barrier integrity and function in a murine model. J Biol Chem 2011; 286:26667-79.

11 Post S, Nawijn MC, Hackett TL et al. The composition of house dust mite is critical for mucosal barrier dysfunction and allergic sensitisation. Thorax 2012; 67:488-95.
12 Piyadasa H, Altieri A, Basu S, Schwartz J, Halayko AJ, Mookherjee N. Biosignature for airway inflammation in a house dust mite-challenged murine model of allergic asthma. Biol Open 2016; 5:112-21.

13 Miglino N, Roth M, Tamm M, Borger P. House dust mite extract downregulates $\mathrm{C} / \mathrm{EBP} \alpha$ in asthmatic bronchial smooth muscle cells. Eur Respir J $2011 ; 38: 50-8$.

14 Buzney CD, Gottlieb AB, Rosmarin D. Asthma and atopic dermatitis: a review of targeted inhibition of interleukin-4 and interleukin-13 as therapy for atopic disease. J Drugs Dermatol 2016; 15:165-71.

15 Porsbjerg C, Baines K, Gibson P et al. IL-33 is related to innate immune activation and sensitization to HDM in mild steroid-free asthma. Clin Exp Allergy 2016; 46:564-74.

16 Sallmann E, Reininger B, Brandt S et al. High-affınity IgE receptors on dendritic cells exacerbate Th2-dependent inflammation. J Immunol 2011; 187:164-71.

17 Mathie SA, Dixon KL, Walker SA et al. Alveolar macrophages are sentinels of murine pulmonary homeostasis following inhaled antigen challenge. Allergy 2015; 70:80-9.

18 Herbert C, Scott MM, Scruton KH et al. Alveolar macrophages stimulate enhanced cytokine production by pulmonary CD4+ T-lymphocytes in an exacerbation of murine chronic asthma. Am J Pathol 2010; 177:165764.

19 Thornadtsson A, Neerincx AH, Hogman $\mathrm{M}$ et al. Extended nitric oxide analysis may improve personalized anti-inflammatory treatment in asthmatic children with intermediate $\mathrm{F}(\mathrm{E}) \mathrm{NO50}$. $J$ Breath Res 2015; 9:047114.

20 Robinson DS. The role of the T cell in asthma. J Allergy Clin Immunol 2010; 126:1081-91; quiz 92-3.

21 Eusebio M, Kuna P, Kraszula L, Kupczyk M, Pietruczuk M. Allergy-related changes in levels of CD8+CD25+FoxP3 (bright) Treg cells and FoxP3 mRNA expression in peripheral blood: the role of IL-10 or TGF-beta. J Biol Regul Homeost Agents 2014; 28:461-70.
22 Farahani R, Sherkat R, Hakemi MG, Eskandari N, Yazdani R. Cytokines (interleukin-9, IL-17, IL-22, IL-25 and IL33) and asthma. Adv Biomed Res 2014; 3:127.

23 Li BW, de Bruijn MJ, Tindemans I et al. T cells are necessary for ILC2 activation in house dust mite-induced allergic airway inflammation in mice. Eur J Immunol 2016; 46:1392-403.

24 Aboud OA, Weiss RH. New opportunities from the cancer metabolome. Clin Chem 2013; 59:138-46.

25 Guasch-Ferré M, Hruby A, Toledo E et al. Metabolomics in prediabetes and diabetes: a systematic review and meta-analysis. Diabetes Care 2016; 39:833-46.

26 Bystrom J, Amin K, Bishop-Bailey D. Analysing the eosinophil cationic protein-a clue to the function of the eosinophil granulocyte. Respir Res 2011; 12:10.

27 Vijverberg SJ, Hilvering B, Raaijmakers JA, Lammers JW, Maitland-van der Zee AH, Koenderman L. Clinical utility of asthma biomarkers: from bench to bedside. Biologics 2013; 7:199-210.

28 Page S, Ammit AJ, Black JL, Armour CL. Human mast cell and airway smooth muscle cell interactions: implications for asthma. Am J Physiol Lung Cell Mol Physiol 2001; 281:L1313-23.

29 Amin K, Janson C, Boman G, Venge P. The extracellular deposition of mast cell products is increased in hypertrophic airways smooth muscles in allergic asthma but not in nonallergic asthma. Allergy 2005; 60:1241-7.

30 Amin K. The role of mast cells in allergic inflammation. Respir Med 2012; 106:9-14.

31 Mo LH, Yang LT, Zeng L et al. Dust mite allergen, glutathione S-transferase, induces $\mathrm{T}$ cell immunoglobulin mucin domain-4 in dendritic cells to facilitate initiation of airway allergy. Clin Exp Allergy 2017; 47:264-270.

32 Piacentini S, Polimanti R, Moscatelli B, Re MA, Manfellotto D, Fuciarelli M. Lack of association between GSTM1, GSTP1, and GSTT1 gene polymorphisms and asthma in adult patients from Rome, central Italy. J Investig Allergol Clin Immunol 2012; 22:252-6. 
33 Sohn SW, Jung JW, Lee SY et al. Expression pattern of GSTP1 and GSTA1 in the pathogenesis of asthma. Exp Lung Res 2013; 39:173-81.

34 Wang IJ, Tsai CH, Chen CH, Tung KY, Lee YL. Glutathione S-transferase, incense burning and asthma in children. Eur Respir J 2011; 37:1371-7.

35 Tripathi P, Awasthi S, Husain N, Prasad R, Mishra V. Increased expression of ADAM33 protein in asthmatic patients as compared to non-asthmatic controls. Indian J Med Res 2013; 137:507-14.

36 Yang IV, Pedersen BS, Liu A et al. DNA methylation and childhood asthma in the inner city. $J$ Allergy Clin Immunol 2015; 136:69-80.

37 Harb H, Raedler D, Ballenberger N et al. Childhood allergic asthma is associated with increased IL-13 and FOXP3 histone acetylation. J Allergy Clin Immunol 2015; 136:200-2.

38 Wegienka G, Havstad S, Kim H et al. Subgroup differences in the associations between dog exposure during the first year of life and early life allergic outcomes. Clin Exp Allergy 2016; 47:97-105.

39 Joubert BR, Felix JF, Yousefi $\mathrm{P}$ et al. DNA methylation in newborns and maternal smoking in pregnancy: genome-wide consortium meta-analysis. Am J Hum Genet 2016; 98:68096.

40 Stokholm J, Sevelsted A, Bonnelykke $\mathrm{K}$, Bisgaard H. Maternal propensity for infections and risk of childhood asthma: a registry-based cohort study. Lancet Respir Med 2014; 2:631-7.

41 Beckhaus AA, Garcia-Marcos L, Forno E, Pacheco-Gonzalez RM, Celedon JC, Castro-Rodriguez JA. Maternal nutrition during pregnancy and risk of asthma, wheeze, and atopic diseases during childhood: a systematic review and meta-analysis. Allergy 2015; 70:1588-604.

42 Ho SM. Environmental epigenetics of asthma: an update. $J$ Allergy Clin Immunol 2010; 126:453-65.

43 House JS, Wyss AB, Hoppin JA et al. Early-life farm exposures and adult asthma and atopy in the agricultural lung health study. $J$ Allergy Clin Immunol 2016; Epub ahead of print.

44 Mackenzie KJ, Anderton SM, Schwarze J. Viral respiratory tract infections and asthma in early life: cause and effect? Clin Exp Allergy 2014; 44:9-19.
45 Korevaar DA, Westerhof GA, Wang J et al. Diagnostic accuracy of minimally invasive markers for detection of airway eosinophilia in asthma: a systematic review and meta-analysis. Lancet Respir Med 2015; 3:290-300.

46 Petsky HL, Kew KM, Turner C, Chang AB. Exhaled nitric oxide levels to guide treatment for adults with asthma. Cochrane Database Syst Rev 2016; 9:CD011440.

47 Wagener AH, de Nijs SB, Lutter R et al. External validation of blood eosinophils, $\mathrm{FE}(\mathrm{NO})$ and serum periostin as surrogates for sputum eosinophils in asthma. Thorax 2015; 70:115-20.

48 Jia G, Erickson RW, Choy DF et al. Periostin is a systemic biomarker of eosinophilic airway inflammation in asthmatic patients. $J$ Allergy Clin Immunol 2012; 130:647-54.e10.

49 Chang C, Guo ZG, He B, Yao WZ. Metabolic alterations in the sera of Chinese patients with mild persistent asthma: a GC-MS-based metabolomics analysis. Acta Pharmacol Sin 2015; 36:1356-66.

50 Ried JS, Baurecht H, Stückler F et al. Integrative genetic and metabolite profiling analysis suggests altered phosphatidylcholine metabolism in asthma. Allergy 2013; 68:629-36.

51 Louhelainen N, Myllärniemi M, Rahman I, Kinnula VL. Airway biomarkers of the oxidant burden in asthma and chronic obstructive pulmonary disease: current and future perspectives. Int $J$ Chron Obstruct Pulmon Dis 2008; 3:585-603.

52 Leung TF, Ko FW, Wong GW. Recent advances in asthma biomarker research. Ther Adv Respir Dis 2013; 7:297-308.

53 Ho WE, Xu YJ, Cheng C et al. Metabolomics reveals inflammatory-linked pulmonary metabolic alterations in a murine model of house dust miteinduced allergic asthma. J Proteome Res 2014; 13:3771-82.

54 Carraro S, Giordano G, Reniero F et al. Asthma severity in childhood and metabolomic profiling of breath condensate. Allergy 2013; 68:110-7.

55 Gahleitner F, Guallar-Hoyas C, Beardsmore CS, Pandya HC, Thomas CP. Metabolomics pilot study to identify volatile organic compound markers of childhood asthma in exhaled breath. Bioanalysis 2013; 5:2239-47.

56 Fens N, de Nijs SB, Peters S et al. Exhaled air molecular profiling in relation to inflammatory subtype and activity in COPD. Eur Respir J 2011; 38:1301-9.

57 Motta A, Paris D, D'Amato $\mathrm{M}$ et al. NMR metabolomic analysis of exhaled breath condensate of asthmatic patients at two different temperatures. J Proteome Res 2014; 13:6107-20.

58 Sinha A, Krishnan V, Sethi $\mathrm{T}$ et al. Metabolomic signatures in nuclear magnetic resonance spectra of exhaled breath condensate identify asthma. Eur Respir J 2012; 39:500-2.

59 Carraro S, Rezzi S, Reniero F et al. Metabolomics applied to exhaled breath condensate in childhood asthma. Am J Respir Crit Care Med 2007; 175:986-90.

60 van der Schee MP, Palmay R, Cowan J0, Taylor DR. Predicting steroid responsiveness in patients with asthma using exhaled breath profiling. Clin Exp Allergy 2013; 43:1217-25.

61 Fens N, Roldaan AC, van der Schee MP et al. External validation of exhaled breath profiling using an electronic nose in the discrimination of asthma with fixed airways obstruction and chronic obstructive pulmonary disease. Clin Exp Allergy 2011; 41:1371-8.

62 Bos LD, Sterk PJ, Fowler SJ. Breathomics in the setting of asthma and chronic obstructive pulmonary disease. J Allergy Clin Immunol 2016; 138:970-6.

63 Rufo JC, Madureira J, Fernandes EO, Moreira A. Volatile organic compounds in asthma diagnosis: a systematic review and meta-analysis. Allergy 2016; 71:175-88.

64 Reisdorph N, Wechsler ME. Utilizing metabolomics to distinguish asthma phenotypes: strategies and clinical implications. Allergy 2013; 68:959-62.

65 Amaral AF. Metabolomics of asthma. $J$ Allergy Clin Immunol 2014; 133:14979, 99.e1.

66 Adamko DJ, Sykes BD, Rowe BH. The metabolomics of asthma: novel diagnostic potential. Chest 2012; 141:1295-302.

67 Scrivo R, Casadei L, Valerio M, Priori R, Valesini G, Manetti C. Metabolomics approach in allergic and rheumatic diseases. Curr Allergy Asthma Rep 2014; 14:445.

68 Kim MA, Shin YS, Pham LD, Park HS. Adult asthma biomarkers. Curr Opin Allergy Clin Immunol 2014; 14:49-54.

69 Nobakht MGhBF, Aliannejad R, Rezaei-Tavirani M, Taheri S, Oskouie 
AA. The metabolomics of airway diseases, including COPD, asthma and cystic fibrosis. Biomarkers 2015; 20:5-16.

70 Snowden S, Dahlén SE, Wheelock CE. Application of metabolomics approaches to the study of respiratory diseases. Bioanalysis 2012; 4:2265-90.

71 Auffray C, Adcock IM, Chung KF, Djukanovic R, Pison C, Sterk PJ. An integrative systems biology approach to understanding pulmonary diseases. Chest 2010; 137:1410-6.

72 Atzei A, Atzori L, Moretti C et al. Metabolomics in paediatric respiratory diseases and bronchiolitis. J Matern Fetal Neonatal Med 2011; 24(Suppl. 2):59-62.

73 Jung J, Kim SH, Lee HS et al. Serum metabolomics reveals pathways and biomarkers associated with asthma pathogenesis. Clin Exp Allergy 2013; 43:425-33.

74 Comhair SA, McDunn J, Bennett C, Fettig J, Erzurum SC, Kalhan SC. Metabolomic endotype of asthma. J Immunol 2015; 195:643-50.

75 Loureiro CC, Duarte IF, Gomes J et al. Urinary metabolomic changes as a predictive biomarker of asthma exacerbation. J Allergy Clin Immunol 2014; 133:261-3.e1-5.

76 Loureiro CC, Duarte IF, Gomes J et al. Unsuspected mild emphysema in nonsmoking patients with chronic asthma with persistent airway obstruction. $J$ Allergy Clin Immunol 2014; 133:2635.e5.

77 Mattarucchi E, Baraldi E, Guillou C. Metabolomics applied to urine samples in childhood asthma; differentiation between asthma phenotypes and identification of relevant metabolites. Biomed Chromatogr 2012; 26:89-94.

78 McGeachie MJ, Dahlin A, Qiu W et al. The metabolomics of asthma control: a promising link between genetics and disease. Immun Inflamm Dis 2015; 3:224-38.

79 Ho WE, Xu YJ, Xu F et al. Metabolomics reveals altered metabolic pathways in experimental asthma. Am J Respir Cell Mol Biol 2013; 48:204-11.

$80 \mathrm{Yu}$ M, Cui FX, Jia HM et al. Aberrant purine metabolism in allergic asthma revealed by plasma metabolomics. $J$ Pharm Biomed Anal 2016; 120:181-9.

81 Saude EJ, Obiefuna IP, Somorjai RL et al. Metabolomic biomarkers in a model of asthma exacerbation: urine nuclear magnetic resonance. $A m \mathrm{~J}$ Respir Crit Care Med 2009; 179:25-34.

82 Saude EJ, Skappak CD, Regush S et al. Metabolomic profiling of asthma: diagnostic utility of urine nuclear magnetic resonance spectroscopy. J Allergy Clin Immunol 2011; 127:757-64.e1-6.

83 Singh A, Yamamoto M, Kam SH et al. Gene-metabolite expression in blood can discriminate allergen- induced isolated early from dual asthmatic responses. PLOS ONE 2013; 8:e67907.

84 Singh A, Shannon CP, Kim YW et al. Identifying molecular mechanisms of the late-phase asthmatic response by integrating cellular, gene, and metabolite levels in blood. Ann Am Thorac Soc 2016; 13(Suppl. 1):S98.

$85 \mathrm{Hu} \mathrm{X}, \mathrm{Wu}$ GP, Zhang MH et al. GCMS-based metabolic profiling reveals metabolic changes in anaphylaxis animal models. Anal Bioanal Chem 2012; 404:887-93.

86 Kong J, Chalcraft K, Mandur TS et al. Comprehensive metabolomics identifies the alarmin uric acid as a critical signal for the induction of peanut allergy. Allergy 2015; 70:495-505.

87 Ibrahim B, Marsden P, Smith JA, Custovic A, Nilsson M, Fowler SJ. Breath metabolomic profiling by nuclear magnetic resonance spectroscopy in asthma. Allergy 2013; 68:1050-6.

88 Chaves Loureiro C, Oliveira AS, Santos $\mathrm{M}$ et al. Urinary metabolomic profiling of asthmatics can be related to clinical characteristics. Allergy 2016; 71:13625.

89 Adamko DJ, Nair P, Mayers I, Tsuyuki RT, Regush S, Rowe BH. Metabolomic profiling of asthma and chronic obstructive pulmonary disease: a pilot study differentiating diseases. $J$ Allergy Clin Immunol 2015; 136:571-80.e3. 